Ciência Florestal, Santa Maria, v. 24, n. 1, p. 59-69, jan.-mar., 2014

ISSN 0103-9954

\title{
ESTOQUE DE CARBONO NO SOLO SOB DIFERENTES FORMAÇÕES FLORESTAIS, CHAPECÓ - SC
}

\section{CARBON STOCK IN SOIL UNDER DIFFERENT FOREST FORMATIONS, CHAPECÓ, SANTA CATARINA STATE}

\author{
Rosiane Berenice Nicoloso Denardin ${ }^{1}$ Jorge Luis Mattias ${ }^{2}$ Leandro do Prado Wildner ${ }^{3}$ \\ Cristiano Nunes Nesi ${ }^{3}$ André Sordi ${ }^{4}$ Daniel Fernando Kolling ${ }^{4}$ Fábio José Busnello ${ }^{4}$ Tadeu Cerutti ${ }^{4}$ \\ RESUMO
}

A adoção de práticas de manejo que garantam a estabilidade da matéria orgânica do solo (MOS) também sustenta a estabilidade ou o incremento quantitativo do carbono (C) na litosfera, diminuindo a quantidade do $\mathrm{CO}_{2}$ na atmosfera. Também é possível minimizar as perdas de $\mathrm{C}$ para a atmosfera a partir da utilização de práticas conservacionistas, ou utilização de coberturas vegetais que mantenham os estoques de $\mathrm{C}$ do solo, sendo que as coberturas florestais são consideradas grandes sequestradoras e os sistemas florestais considerados grandes reservatórios de C. Este trabalho foi realizado em uma propriedade rural situada no interior do município de Chapecó-SC, onde foram amostrados solos de diferentes formações florestais distribuídas em uma faixa homogênea do solo. O clima do local é mesotérmico, chuvoso, e o solo foi caracterizado como uma associação Cambissolo Háplico/Neossolo Litólico. Os objetivos deste trabalho foram estimar os estoques de $\mathrm{C}$ nos solos e as perdas de $\mathrm{C}$ ocorridas em função da alteração da cobertura do solo. Foram avaliados solos sob floresta natural (FN), de formação secundária, com elevado grau de preservação; plantio de eucalipto (Eucalyptus saligna) (PE), com oito anos de cultivo, precedido de 17 anos de lavoura sob plantio convencional; plantio de erva-mate (Ilex paraguariensis) (EM), com 25 anos de cultivo sob sistema convencional (corte em intervalo de 18 meses, com retirada de todos resíduos produzidos e manutenção do solo sem cobertura, com utilização periódica de herbicida - glifosato). Em cada área foram abertas quatro trincheiras de $50 \mathrm{~cm}$ de profundidade, onde foram realizadas as coletas de solo nas profundidades de: $0-5 \mathrm{~cm} ; 5-10 \mathrm{~cm} ; 10-20 \mathrm{~cm} ; 20-30 \mathrm{~cm} ; 30-40 \mathrm{~cm}$, e 40-50 cm, com anéis de kopeck. Foi possível determinar a densidade do solo $\left(\mathrm{Mg} \mathrm{m}^{-3}\right)$ e o volume do solo por camada (profundidade) e por hectare, bem como a concentração de $\mathrm{C}$ do solo nas diferentes áreas estudadas. Para quantificar os estoques de $\mathrm{C}$ foram utilizadas quantidades iguais de solo para cada profundidade avaliada. Foram observadas maiores densidades dos solos sob PE e EM, sendo que em FN os menores valores de densidade são explicados pelo grande aporte de material orgânico e ausência de efeito antrópico. Em FN, apesar da menor densidade do solo, verificou-se maior estoque de C no solo, com 107,67 $\mathrm{Mg} \mathrm{C}^{-1}$. No solo sob PE, com estoque de 79,58 M g C ha-1, em função da utilização anterior (17 anos com lavoura sob cultivo convencional), supõe-se que parte do C tenha sido recuperada. Sob EM, com estoque de 47,29 $\mathrm{Mg} \mathrm{C} \mathrm{ha}^{-1}$, as perdas de $\mathrm{C}$ foram evidentes, com cerca de $221 \mathrm{Mg} \mathrm{CO}_{2}$ ha $^{-1}$ emitidas do solo. Ficou evidente que a alteração da cobertura florestal e o manejo utilizado podem levar a grandes perdas do C estocado. Assim, o solo sob florestas, ou o sistema solo-planta de coberturas florestais, considerado um grande reservatório de C, pode tornar-se uma grande fonte de $\mathrm{C}$ para a atmosfera, contribuindo para aumentar o efeito estufa.

Palavras-chave: Eucalyptus sp.; Ilex paraguariensis; carbono do solo; quantificação de carbono.

\section{ABSTRACT}

The adoption of management practices that ensure the stability of soil organic matter also maintain the

1 Agrônoma, Dr $^{\mathrm{a}}$., Professora Adjunta do Curso de Agronomia, Universidade Federal da Fronteira Sul, Caixa Postal 181, CEP 89812-000, Chapecó (SC), Brasil. denardin500@gmail.com

2 Agrônomo, Dr., Pesquisador do Cepaf/Epagri, Caixa Postal 791, CEP 898801-970, Chapecó (SC), Brasil. jlmattias@gmail.com

3 Agrônomo, Msc., Pesquisador do Cepaf/Epagri, Caixa Postal 791, CEP 898801-970, Chapecó (SC), Brasil. leandrowildner@gmail.com / cristiano@epagri.sc.gov.br

4 Acadêmicos do Curso de Agronomia, Bolsistas FAPE/Unochapecó, Unochapecó, Caixa Postal 1141, CEP 898809-000, Chapecó (SC), Brasil. andresordi@yahoo.com.br / dzlpolenta@gmail.com / fbusnello@yahoo.com.br / tadeucerutti@gmail.com

Recebido para publicação em 13/04/2010 e aceito em 1/10/2012 
stability or quantitative increase of carbon (C) in the lithosphere, reducing the amount of $\mathrm{CO}_{2}$ in the atmosphere. You can also minimize the losses of $\mathrm{C}$ to the atmosphere by using conservation practices, or using cover crops to keep the soil $\mathrm{C}$ stocks, and the forest cover are considered great abduction and forest systems considered large reservoirs of $\mathrm{C}$. This work was performed on a property located in Chapecó, Santa Catarina state, where soils were sampled from different forest formations distributed in a homogeneous soil range. The local climate is mesothermal, rainy, and the soil was characterized as an association Cambissolo Háplico/Neossolo Litólico. The objectives were to estimate the $\mathrm{C}$ stocks in soils and estimate the $\mathrm{C}$ losses occurred due to the change of soil cover. It was evaluated soils under natural forest (FN), of secondary stage, with a high degree of preservation; planted forest of eucalyptus (Eucalyptus saligna) (PE), with eight years of cultivation, preceded by 17 years under crop conventional tillage; and a planted forest of herb mate (Ilex paraguariensis) (EM), with 25 years of cultivation under conventional system (cutting interval of 18 months, with removal of all waste produced and maintenance of the ground without cover, with periodic use of herbicide - glyphosate). In each area were opened four trenches with $50 \mathrm{~cm}$ deep, where soil samples were collected in depths of: 0-5 cm, 5-10 cm, 10-20 cm, $20-30 \mathrm{~cm}, 30-40 \mathrm{~cm}$, and 40-50 cm, with kopeck rings. It was possible to determine the bulk density $(\mathrm{Mg}$ $\mathrm{m}^{-3}$ ), the soil volume per layer (depth) and per hectare, and the concentration of soil C in the different studied areas. To quantify the $\mathrm{C}$ stocks equal amounts of soil were used for each depth evaluated. It was observed higher densities of soils and under PE and EM, to FN the lowest density are explained by the large amount of organic material and the absence of anthropogenic effects. In FN, despite the lower bulk density, there was a greater soil content $\mathrm{C}$, with $107.67 \mathrm{Mg} \mathrm{C} \mathrm{ha}^{-1}$. On the soil under PE, with stock $79.58 \mathrm{Mg} \mathrm{C} \mathrm{ha}^{-1}$, depending on previous use (17 years under crop conventional tillage), it is assumed that part of $\mathrm{C}$ has been recovered. Under EM, with stock of 47.29 $\mathrm{Mg} \mathrm{C}^{-1}$, C losses were evident, with about $221 \mathrm{Mg} \mathrm{CO}_{2} \mathrm{ha}^{-1}$ emitted from the soil. It was evident that the change in forest cover and management procedures can lead to large losses of $\mathrm{C}$ stored. Thus, the soil under forest, or the soil-plant a forest canopy, considered a major reservoir of $\mathrm{C}$, can become a major source of $\mathrm{C}$ to the atmosphere, contributing to increased the greenhouse effect.

Keywords: Eucalyptus sp.; Ilex paraguariensis; soil carbon; carbon quantification.

\section{INTRODUÇÃO}

Os estudos do ciclo do carbono (C) apresentam grande interesse devido à sua implicação nas mudanças climáticas, por influência da alteração das emissões dos gases de efeito estufa (GEEs). As práticas agrícolas e as mudanças do uso do solo devido ao desmatamento são as principais fontes de emissão dos GEEs. O Brasil está situado em $5^{\circ}$ lugar na classificação mundial dos países emissores destes gases, considerando essas ações antrópicas, sendo que aproximadamente $75 \%$ do $\mathrm{CO}_{2}$ emitido para a atmosfera são derivados de práticas agrícolas inadequadas e do desmatamento e apenas $18 \%$ são originados da queima de combustíveis fósseis (MCT, 2010).

Assim como nas demais regiões do Brasil, em todo o sul do país, grandes áreas de florestas naturais foram desmatadas devido ao aumento da necessidade de áreas para produção agrícola, restando pequenos remanescentes de matas onde o $\mathrm{C}$ cumpre de forma mais estanque o seu ciclo.
As plantas fixam $\mathrm{C}$ via fotossíntese, a partir do $\mathrm{CO}_{2}$ atmosférico, que passa a constituir suas moléculas estruturais e funcionais. Os ecossistemas terrestres acumulam $\mathrm{C}$ no decorrer da sucessão, porém, este acúmulo declina enquanto as florestas atingem a maturidade (SCHOLES e NOBLE, 2000). As florestas representam um potencial de mitigação temporário, porém, significativo, pois até 2050 , serão fixadas 60-87 Gt C ano ${ }^{-1}$ nas florestas e $23-44 \mathrm{Gt} \mathrm{C}$ ano $^{-1}$ nos solos agrícolas (IPCC, 2001).

$\mathrm{O}$ conceito de fixação de $\mathrm{C}$ normalmente se relaciona com a ideia de armazenar $\mathrm{C}$ em solos, florestas e outros tipos de vegetação (BALBINOT et al., 2003), contudo, a retirada e/ou a substituição da vegetação altera estes reservatórios e sumidouros. Ao contrário, as alterações da vegetação em cobertura e do solo podem tornar um ecossistema dreno de $\mathrm{C}$ em fonte de C para a atmosfera (BAYER et al., 2004; DIEKOW et al., 2005).

A entrada de C orgânico no solo depende da entrada de material orgânico através da senes- 
cência de certos componentes da biomassa acima e abaixo do solo, queda das folhas, resíduos da exploração e animais mortos, com suas respectivas taxas de decomposição. A deposição e a subsequente decomposição da fitomassa é o principal caminho para a transferência de $\mathrm{C}$ e nutrientes da planta para o solo (SCHUMACHER et al., 2004). Neste sentido, ressalta-se o papel das florestas naturais e dos plantios florestais como ferramentas úteis de sequestro e fixação de $\mathrm{C}$, podendo influenciar a sua distribuição nos diferentes componentes do material orgânico do ecossistema, tais como solo, serapilheira, raízes e biomassa (COSTA et al., 2005).

Entretanto, faz-se urgente avaliar o potencial de acúmulo de $\mathrm{C}$ e as possíveis alterações nas suas perdas no solo sob diferentes coberturas florestais, pois a quantidade média de $\mathrm{C}$ orgânico estocado no solo varia muito em função do tipo de floresta ou povoamento que o ocupa (CALDEIRA et al., 2003). Tornquist et al. (2005) reportam que a dinâmica do $\mathrm{C}$ nos ecossistemas requer o estabelecimento de estoques iniciais de referência, existentes no solo antes da intervenção antrópica, a partir dos quais são verificados os impactos do uso e manejo dos solos. Estes estoques originais são também considerados indicativos do potencial de armazenagem de $\mathrm{C}$ em cada classe/tipo de solo.

Assim, este trabalho objetivou avaliar o potencial de acúmulo de $\mathrm{C}$ no solo sob diferentes formações florestais (FFs): plantio de eucalipto (Eucalyptus saligna) (PE), plantio de erva-mate (Ilex paraguariensis) (EM) e floresta natural (FN), no município de Chapecó, região Oeste de Santa Catarina, sendo a mata nativa a área de referência a ser utilizada.

\section{MATERIAL E MÉTODO}

O presente estudo foi realizado em propriedade particular localizada na comunidade de Colônia Bacia, município de Chapecó, região Oeste do Estado de Santa Catarina, nos meses de junho e julho de 2008. A propriedade encontra-se nas coordenadas geográficas $26^{\circ} 59^{\prime} 41^{\prime \prime} \mathrm{S}$ e $52^{\circ} 42^{\prime} 47^{\prime \prime} \mathrm{O}$, com altitude média de $670 \mathrm{~m}$. De acordo com a classificação climática de Köppen, a região apresenta clima do tipo mesotérmico úmido, verão quente [Cfa] (PANDOLFO, 2001), com temperaturas máximas médias de $23^{\circ} \mathrm{C}$ e mínimas médias de $14,0^{\circ} \mathrm{C}$. A precipitação média anual da região é de $2.039 \mathrm{~mm}$ (dados médios fornecidos pela Estação Meteorológica da EPAGRI/ CEPAF, Chapecó-SC). Os solos da área pertencem à associação Cambissolo Háplico/Neossolo Litólico (EMBRAPA, 2006).

Foram amostradas três áreas contíguas, com diferentes formações florestais (FFs): floresta natural (FN), de formação secundária, com elevado grau de preservação, originalmente como um ecótono ou zona de contato entre duas formações florestais, Floresta Ombrófila Mista (montanha) e Floresta Estacional Decidual (submontanha e montanha) (MUELLERDOMBOIS e ELLENBERG, 1974); plantio de eucalipto (PE), com oito anos de cultivo, precedido de 17 anos de lavoura sob sistema de plantio convencional (PC); plantio de erva-mate (EM), com 25 anos de cultivo sob sistema "tradicional" (cortes em intervalos de 18 meses e manutenção do solo sem cobertura, com utilização periódica de herbicida - glifosato).

Em cada área estudada foram abertas quatro trincheiras de $50 \mathrm{~cm}$ de profundidade, totalizando 12 trincheiras. Nestas, foram realizadas as coletas de solo para determinação da densidade do solo. As amostras foram coletadas nas profundidades: $0-5 \mathrm{~cm}$; $5-10 \mathrm{~cm} ; 10-20 \mathrm{~cm} ; 20-30 \mathrm{~cm} ; 30-40 \mathrm{~cm}$, e $40-50 \mathrm{~cm}$, com anéis de kopeck (EMBRAPA, 1997). As amostras foram acondicionadas e levadas ao Laboratório de Solos da Unochapecó, onde foram secas em estufa $\left(105^{\circ} \mathrm{C}\right)$ até peso constante e, após, pesadas em balanças de precisão. Para os cálculos da densidade do solo $\left(\mathrm{Mg} \mathrm{m}^{-3}\right)$ e volume do solo por camada (profundidade) e por hectare, bem como da concentração de $\mathrm{C}$ do solo nas diferentes áreas sob estudo, utilizou-se o método proposto por Arevalo et al. (2002).

Nas mesmas trincheiras e profundidades também foram coletadas amostras de solo para determinação de C. Além dessas, aleatoriamente, foram coletadas amostras em mais 16 pontos, nas mesmas profundidades, totalizando 20 pontos de amostragem em cada área.

As amostras de solo foram acondicionadas e encaminhadas ao laboratório (Unochapecó). Todas as análises foram feitas em duplicatas, segundo EMBRAPA (1997), adaptada para bloco digestor, com a digestão de $0,5 \mathrm{~g}$ de solo em $10 \mathrm{~mL}$ de dicromato potássico $\left(\mathrm{K}_{2} \mathrm{Cr}_{2} \mathrm{O}_{7}\right) 0,4 \mathrm{~mol} \mathrm{~L}^{-1}$, por 30 minutos a $150{ }^{\circ} \mathrm{C}$. Após resfriada, a amostra era transferida para frascos erlenmeyers de $250 \mathrm{ml}$ adicionando-se $80 \mathrm{~mL}$ de água e três gotas de indicador difenilamina com posterior titulação com sulfato ferroso $\left(\mathrm{Fe}\left(\mathrm{NH}_{4}\right)_{2}\left(\mathrm{SO}_{4}\right)_{2} \cdot 6 \mathrm{H}_{2} \mathrm{O}\right) \quad 0,1 \mathrm{~mol} \mathrm{~L}^{-1}$.

Para quantificar os estoques de $\mathrm{C}$ nos diferentes FFs foi utilizado o método proposto por Sisti et al. (2004), com quantidades iguais de solo para cada profundidade avaliada. Para corrigir a massa do solo, 
diferente naturalmente em cada uma das profundidades e deixá-la igual nas diferentes áreas avaliadas, permitindo a comparação, a metodologia propõe que sejam subtraídas as quantidades de $\mathrm{C}$ das maiores profundidades do solo amostrado $(40-50 \mathrm{~cm})$, da mesma massa da profundidade da FF de referência, no caso a $\mathrm{FN}$, quando as massas de solos não são equivalentes, conforme a equação que segue:

$$
C s=\sum_{i=1}^{n-1} C T i+\left[M T n-\left(\sum_{i=1}^{n} M T i-\sum_{i=1}^{n} M S i\right)\right] \times C T n
$$

Em que:

$C_{S}$ é o estoque de C total no solo $\left(\mathrm{Mg} \mathrm{Cha}^{-1}\right)$, em uma profundidade onde a massa de solo é a mesma daquela observada no perfil de solo da área referência $(\mathrm{FN})$;

$$
\sum_{i=1}^{n-1} C T i \text { é a soma do conteúdo de } \mathrm{C} \text { total }
$$

$\left(\mathrm{Mg} \mathrm{ha}^{-1}\right)$ da camada superficial até a penúltima camada de solo com outros FF (PE e EM);

$$
\sum_{i=1}^{n} M S i \text { é a massa de solo }\left(\mathrm{Mg} \mathrm{ha}^{-1}\right) \text { da ca- }
$$
mada superficial até a última camada do solo da área de referência (FN);

$$
\sum_{i=1}^{n} M T i \text { é a soma da massa de solo }\left(\mathrm{Mg} \mathrm{ha}^{-1}\right)
$$
da camada superficial até a última camada do solo sob tratamentos com diferentes FF (PE e EM);

$\boldsymbol{M}_{\mathrm{Tn}}$ e $\boldsymbol{C}_{\mathrm{Tn}}$ são, respectivamente, a massa de solo $(\mathrm{Mg})$ e a concentração de $\mathrm{C}(\mathrm{Mg} \mathrm{C} \mathrm{Mg} \mathrm{solo-1)}$ na última camada do perfil do solo com diferentes FF (PE e EM).

A quantidade de $\mathrm{C}$ emitida em forma de $\mathrm{CO}_{2}$ nos diferentes $\mathrm{SF}$ foi estimada segundo Spagnollo (2004), que utiliza as perdas de C das áreas analisadas na forma de $\mathrm{CO}_{2}$, utilizando iguais pesos moleculares para ambos os elementos.

Os dados de densidade do solo $\left(\mathrm{Mg} \mathrm{m}^{-3}\right) \mathrm{e}$ teor de $\mathrm{C}$ do solo $\left(\mathrm{g} \mathrm{kg}^{-1}\right)$, das três FFs foram submetidos à análise de variância e ao teste de Tukey, considerando probabilidade de erro de $1 \%(\mathrm{P}>0,01)$, utilizando-se o Statistical Analysis System (SAS).

\section{RESULTADOS E DISCUSSÃO}

\section{Densidade do solo}

Nas três áreas avaliadas verificou-se menor densidade na camada superficial do solo. Isso pode ser explicado por ser a camada de maior interação com a fonte de resíduos, ou ser a camada de maior aporte de resíduos orgânicos. Ainda assim, em PE e EM, não se verificou diferença significativa entre as diferentes profundidades. Já em FN, esta diferença foi evidente $(\mathrm{P}<0,01)$ (Tabela 1).

Em FN, a variação da densidade apresentou o padrão que normalmente se verifica para a maioria dos solos, isto é, em condições naturais, aumenta em profundidade, em função da diminuição do teor de matéria orgânica (MO) e do peso das camadas de solo sobrejacentes (CAVENAGE et al., 1999). A média de densidade para o solo de FN foi a menor entre as áreas estudadas, com valor de $0,96 \mathrm{Mg} \mathrm{m}^{-3}$, que pode ser devido à maior quantidade de resíduos orgânicos sobre o solo. A maior densidade nas áreas manejadas (PE e EM) também pode estar relacionada ao menor aporte de material orgânico ao longo do período. Segundo Suzuki et al. (2002) e Araújo et al. (2004), a menor densidade encontrada em solo sob mata nativa pode ser atribuída ao maior e contínuo aporte de resíduos orgânicos, bem como a não ocorrência de ação antrópica que provoque compactação (revolvimento do solo, tráfego de máquinas).

A manutenção da cobertura vegetal em áreas de floresta garante um grande aporte de resíduos tanto sobre o solo quanto sob o solo (raízes). Em $\mathrm{PE}$, apesar do alto aporte de resíduos orgânicos (verificou-se considerável acúmulo de serapilheira), o solo apresentou os maiores valores de densidade. Pode-se inferir que, apesar dos oito anos de cultivo do eucalipto e não revolvimento do solo, este período não foi suficiente para recuperação das caracte-

TABELA 1: Densidade do solo $\left(\mathrm{Mg} \mathrm{m}^{-3}\right)$, em diferentes profundidades e formações

\begin{tabular}{|c|c|c|c|}
\hline \multicolumn{4}{|c|}{ Densidade do Solo $\mathrm{Mg} \mathrm{m}^{-3}$} \\
\hline $\begin{array}{l}\text { Profundidade } \\
(\mathrm{cm})\end{array}$ & $\begin{array}{l}\text { Floresta } \\
\text { Natural }\end{array}$ & Eucalipto & Erva-Mate \\
\hline $0-5$ & $0,75 \mathrm{~A} \quad \mathrm{~b}$ & $1,10 \mathrm{~A} \mathrm{a}$ & $0,99 \mathrm{~A} \mathrm{ab}$ \\
\hline $5-10$ & $0,89 \mathrm{AB} \quad \mathrm{b}$ & $1,24 \mathrm{~A} \mathrm{a}$ & $1,01 \mathrm{~A} \mathrm{ab}$ \\
\hline $10-20$ & $0,95 \mathrm{AB} \quad \mathrm{b}$ & $1,24 \mathrm{~A} \mathrm{a}$ & $0,98 \mathrm{~A} \mathrm{ab}$ \\
\hline $20-30$ & $1,01 \mathrm{AB}$ a & $1,22 \mathrm{~A} \mathrm{a}$ & $1,01 \mathrm{~A} \mathrm{a}$ \\
\hline $30-40$ & $1,04 \quad \mathrm{~B} \mathrm{a}$ & $1,20 \mathrm{~A} \mathrm{a}$ & $1,01 \mathrm{~A} \mathrm{a}$ \\
\hline $40-50$ & $1,07 \quad \mathrm{~B} \mathrm{a}$ & $1,20 \mathrm{~A} \mathrm{a}$ & $1,01 \mathrm{~A} \mathrm{a}$ \\
\hline
\end{tabular}
florestais.

TABLE 1: Bulk density $\left(\mathrm{Mg} \mathrm{m}^{-3}\right)$, at different depths and forest formations.

Em que: médias seguidas por letras maiúsculas iguais na coluna e médias seguidas por letras minúsculas iguais na linha não diferem entre si pelo teste de Tukey ao nível de $1 \%$ de significância. 
rísticas originais do solo (solo da FN). Pois há que se considerar que a área esteve sob cultivo de espécies anuais (soja, aveia e milho) em plantio convencional durante 17 anos.

No solo sob EM, também se verificou aumento da densidade, nas camadas mais superficiais, apesar de não diferir significativamente $(\mathrm{P}>0,01)$ do solo referência (FN). Ao contrário do solo sob $\mathrm{PE}$, o solo sob EM somente foi manipulado por ocasião da retirada da FN e introdução (plantio) da erva-mate. Desta forma, atribui-se o aumento da densidade do solo em EM à drástica redução do aporte de material orgânico, bem como à manutenção do solo sem cobertura, visto que a área foi mantida "limpa", através do uso sistemático de herbicidas, não sendo permitido o crescimento e/ou estabelecimento de espécies espontâneas.

\section{Teor de Carbono no solo}

De um modo geral, verifica-se padrão característico para o tipo de solo estudado, com os diferentes usos, ou seja, com maiores concentrações nas camadas superficiais e diminuição dos teores de $\mathrm{C}$ com o aumento da profundidade em todas as áreas avaliadas $(\mathrm{P}<0,01)$ (Tabela 2$)$, visto que a camada superficial do solo é onde a deposição de material orgânico ocorre com maior intensidade, corroborando com os resultados encontrados por Neves et al. (2004) e Sisti et al. (2004). Estes valores estão diretamente associados ao maior acúmulo de sera-

TABELA 2: Teor de carbono do solo $\left(\mathrm{g} \mathrm{kg}^{-1}\right)$, em diferentes profundidades e formações florestais.

TABLE 2: Carbon content of soil $\left(\mathrm{g} \mathrm{kg}^{-1}\right)$ at different depths and forest formations.

\begin{tabular}{|c|c|c|c|}
\hline \multicolumn{4}{|c|}{ Teor de Carbono $\left(\mathrm{g} \mathrm{kg}^{-1}\right)$} \\
\hline $\begin{array}{l}\text { Profundidade } \\
\text { (m) }\end{array}$ & $\begin{array}{l}\text { Floresta } \\
\text { Natural }\end{array}$ & Eucalipto & Erva-Mate \\
\hline $0-5$ & $45,59 \mathrm{~A} \mathrm{a}$ & $23,53 \mathrm{Ab}$ & $16,95 \mathrm{~A} \mathrm{~b}$ \\
\hline $5-10$ & $35,59 \mathrm{~B}$ a & $19,07 \mathrm{~A} \mathrm{~b}$ & $13,62 \mathrm{~A} b$ \\
\hline $10-20$ & $29,95 \mathrm{~B}$ a & $17,72 \mathrm{AB} b$ & $12,13 \mathrm{AB} b$ \\
\hline $20-30$ & $18,78 \mathrm{C} \mathrm{a}$ & $13,67 \mathrm{BC} a b$ & $10,74 \mathrm{AB} a b$ \\
\hline $30-40$ & $14,11 \mathrm{C}$ a & $11,92 \mathrm{BC} \mathrm{a}$ & $9,21 \mathrm{~B} \mathrm{a}$ \\
\hline $40-50$ & $11,95 \mathrm{C} \mathrm{a}$ & $9,65 \mathrm{C} \mathrm{a}$ & $9,10 \mathrm{~B}$ a \\
\hline
\end{tabular}

Em que: médias seguidas por letras maiúsculas iguais na coluna e médias seguidas por letras minúsculas iguais na linha não diferem entre si pelo teste de Tukey ao nível de $1 \%$ de significância. pilheira e à maior entrada de material orgânico no sistema solo-planta. Com a redução da contribuição da $\mathrm{MO}$ em profundidade, reduz-se também o teor de C (CALDEIRA et al., 2003).

Os resultados demonstram a importância da estabilidade do solo nos sistemas florestais e do aporte de resíduos vegetais. O manejo do solo e a ação de fatores abióticos como radiação, temperatura e umidade influenciam na degradação da $\mathrm{MO}$ e, consequentemente, na concentração de C. Também fica evidente que as maiores concentrações e as maiores perdas de $\mathrm{C}$ ocorrem nas camadas superficiais, sob maior efeito dos microrganismos e dos fatores abióticos, que regulam as atividades destes.

$\mathrm{Na}$ mesma área deste estudo, em pastagens contíguas à área EM, Sordi et al. (2009), comparando áreas de pastagem perene naturalizada (PPN) e pastagem cultivada com espécies anuais (PCA), verificaram que a perda total de $\mathrm{C}(0-50 \mathrm{~cm}) \mathrm{em}$ dois anos de cultivo foi equivalente a $2,34 \mathrm{~g} \mathrm{~kg}^{-1}$ de $\mathrm{C}$, ou seja, 9,65\% do $\mathrm{C}$ original. A maior perda neste sistema foi verificada na camada arável do solo $(0-20 \mathrm{~cm})$, com uma diminuição de $1,98 \mathrm{~g} \mathrm{~kg}^{-1}$ do $\mathrm{C}$ total original do sistema PPN a partir do revolvimento do solo. Nas camadas inferiores os teores foram bastante próximos nas diferentes pastagens.

Costa et al. (2004), em experimento avaliando lavouras em sistema plantio direto (SPD) e plantio convencional (PC), verificaram maior alteração nos estoques de $\mathrm{C}$ nas camadas mais superficiais do solo, sendo que o conteúdo de $\mathrm{C}$ foi $32 \%$ superior no SPD em relação ao PC, atribuindo a isso uma maior adição de resíduos vegetais associada à manutenção destes na superfície do solo, o que diminui a taxa de decomposição microbiana, resultando em um maior acúmulo diferencial, sendo que esta analogia pode ser feita para EM e PE, e levar a supor que, em PE, oito anos após a implantação deste sistema, o solo já recuperou parte do $\mathrm{C}$, provavelmente perdido durante os 17 anos de cultivo convencional. Ao contrário, em EM as perdas foram contínuas durante 25 anos.

Em EM, comparando com os valores encontrados no solo referência ( $\mathrm{FN}$ ), as perdas de C chegaram a $62 \%$ na camada de $0-10 \mathrm{~cm}$ e a $34 \%$ e $24 \%$ nas camadas de 30-40 e 40-50 cm, respectivamente.

Avaliando as alterações na MOS sob diferentes coberturas vegetais, em Missiones-Argentina, Piccolo et al. (2008) relatam uma rápida diminuição da MOS quando a floresta natural foi substituída pela cultura da erva-mate em monocultivo. Os estoques de $\mathrm{C}$ foram reduzidos em cerca de $70 \%$ na 
camada de $0-15 \mathrm{~cm}$. Também reportam que a densidade do solo da floresta, nesta camada, foi $78 \%$ menor. Argumentam que a diminuição de $\mathrm{C}$ e aumento da densidade do solo sob a erva-mate deveram-se ao menor aporte de resíduos vegetais, em função da retirada de galhos e folhas da erva-mate, por ser a matéria-prima desta cultura, bem como pelo contínuo controle mecânico das plantas daninhas (sistema convencional). Este manejo, característico de plantações de erva-mate, promove um intenso tráfego de máquinas sobre um solo com reduzida cobertura. Os autores relatam que as perdas de $\mathrm{C}$ ocorreram não só pela diminuição dos processos biológicos, mas também por erosão. Em parte da área com erva-mate, nas entre linhas, por 10 anos foi cultivado capim-elefante, sendo que neste período o estoque de $\mathrm{C}$ aumentou em 19\%, mas não verificaram alterações na densidade do solo.

Em comparação com outros estudos, os teores de $\mathrm{C}$ para a camada superficial não apresentaram muita variação. Brun (2004) avaliando C no solo de floresta estacional decidual no Rio Grande do Sul encontrou 39,06 $\mathrm{g} \mathrm{kg}^{-1}$ na camada de $0-10 \mathrm{~cm}$ e $10,05 \mathrm{~g} \mathrm{~kg}^{-1}$ na camada mais profunda $(40-50 \mathrm{~cm})$. Souza (2005) encontrou para camada superficial de mata nativa de araucária, $51,7 \mathrm{~g} \mathrm{~kg}^{-1}$, de reflorestamento de araucária com 18 anos, 43,6 $\mathrm{g} \mathrm{kg}^{-1}$, de plantio de Pinus com 20 anos 44,2 $\mathrm{g} \mathrm{kg}^{-1}$ e com 12 anos 47,6 $\mathrm{g} \mathrm{kg}^{-1}$. A autora argumenta que, em geral, a substituição de sistemas naturais por plantios apresenta efeitos na redução da MOS e no C do solo. Já os sistemas naturais caracterizam-se por um estado estacionário, cujas variações nos conteúdos de MO não são observados (ADDISCOTT, 1992). Da mesma forma, conforme o plantio se desenvolve o $\mathrm{C}$ mais superficial aumenta, porém, fica estável em determinada idade e após isto tende a diminuir. Os autores não delimitam até quando este processo pode ocorrer.

Avaliando alterações nas características do solo em função da substituição de cobertura florestal natural por plantações de eucalipto, em diferentes sítios da região sudeste, Gama-Rodrigues et al. (2008) reportam que em solo franco-argiloarenoso (Aracruz-ES) o teor de $\mathrm{C}$ do solo diminuiu de 11,1 para $7,7 \mathrm{~g} \mathrm{~kg}^{-1}$. Em solo argiloso (Guanhães-MG), o teor de $\mathrm{C}$ foi reduzido de 18,4 para $12,3 \mathrm{~g} \mathrm{~kg}^{-1}$. Relatam que a quantidade de serapilheira foi maior nas áreas de eucalipto do que nas formações nativas, mas a redução do $\mathrm{C}$ no solo foi explicada pela maior relação $\mathrm{C} / \mathrm{N}$ na serapilheira do eucalipto.
Andrade (2004), em Itatinga - SP, não observou efeito da aplicação de doses de um biossólido, nos estoques de $\mathrm{C}$ e $\mathrm{N}$ do solo, em um Latossolo cultivado com eucalipto, após cinco anos da aplicação do resíduo. Avaliando as camadas de solo, o autor encontrou $46,58 \mathrm{~g} \mathrm{~kg}^{-1}$ na camada de $0-5 \mathrm{~cm}$ e 9,08 $\mathrm{g} \mathrm{kg}^{-1}$ na camada $20-30 \mathrm{~cm}$, com densidades de 1,09 e $1,41 \mathrm{Mg} \mathrm{m}^{-3}$, respectivamente. Cabe salientar que a área estudada por Andrade (2004) com Eucalyptus grandis, com cinco anos de plantio, foi precedida de cultivo com a mesma espécie, sendo as plantas cortadas com sete anos de idade. Ao contrário da área deste estudo, que foi precedida por lavoura sob PC, durante 17 anos.

No trabalho de Schumacher et al. (2004) em floresta de Pinus elliottii Eng. com 36 anos, verifica-se na profundidade de $0-5 \mathrm{~cm}$ conteúdo de $\mathrm{C}$ no valor de 32,0 $\mathrm{g} \mathrm{kg}^{-1}$, e um estoque de 34,3 $\mathrm{Mg} \mathrm{ha}^{-1}$, considerando a camada $0-20 \mathrm{~cm}$. Os autores relatam que isto se deve à maior quantidade de $\mathrm{MO}$ encontrada nas camadas superficiais e ressaltam que nestes povoamentos é comum uma grande produção e uma lenta decomposição da serapilheira, o que contribui consideravelmente para a elevada concentração de C nos primeiros centímetros do solo nestas florestas.

Diekow (2004) observou perdas de C e N até $100 \mathrm{~cm}$ de profundidade em um solo sob "pousio" por 50 anos, em comparação ao campo nativo, concluindo que as avaliações realizadas somente nas camadas superficiais do solo (até $30 \mathrm{~cm}$ de profundidade) subestimam consideravelmente as perdas reais de $\mathrm{C}$ e $\mathrm{N}$ ocorridas ao longo do perfil. Entretanto, relata que apesar das mudanças dominantes dos estoques de $\mathrm{C}$ ocorrerem principalmente na camada superficial $(0-20 \mathrm{~cm})$, as demais camadas também são importantes reservatórios de $\mathrm{C}$ e, dependendo do manejo adotado, as camadas mais profundas também podem ser fonte ou dreno de $\mathrm{C}$ para a atmosfera (DIEKOW et al., 2005). As mesmas variações e tendências foram constatadas neste trabalho. Ou seja, as maiores perdas verificam-se nas camadas mais superficiais, mas as camadas mais profundas podem contribuir com perdas consideráveis de $\mathrm{C}$, quando se compara o solo da $\mathrm{FN}$ e o solo das áreas antropizadas (PE e EM).

\section{Estoque de Carbono no solo}

Nos dados apresentados na Tabela 3 fica evidente o efeito das diferentes coberturas florestais e do manejo utilizado nas diferentes áreas sobre o estoque atual de $\mathrm{C}$ no solo. 
TABELA 3: Estoque de carbono no solo ( $\mathrm{Mg} \mathrm{C}$ $\left.\mathrm{ha}^{-1}\right)$ em diferentes profundidades $\mathrm{e}$ formações florestais.

TABLE 3: Carbon storage in soil $\left(\mathrm{Mg} \mathrm{C} \mathrm{ha}^{-1}\right)$ at different depths and forest formations.

Estoque de $\mathrm{C}\left(\mathrm{Mg} \mathrm{C} \mathrm{ha}^{-1}\right)$

\begin{tabular}{cccc}
\hline $\begin{array}{c}\text { Profundidade } \\
(\mathrm{cm})\end{array}$ & Mata Nativa & Eucalipto & Erva-Mate \\
\hline $0-5$ & 16,61 & 12,93 & 8,39 \\
$5-10$ & 15,41 & 12,10 & 6,88 \\
$10-20$ & 28,77 & 22,71 & 11,89 \\
$20-30$ & 19,01 & 17,27 & 10,85 \\
$30-40$ & 15,21 & 14,21 & 9,29 \\
$40-50$ & 12,66 & 11,75 & 9,19 \\
Total $^{1}$ & 107,67 & 79,58 & 47,29 \\
\hline
\end{tabular}

Em que: ${ }^{1}=$ Estoque de $\mathrm{C}$, valores calculados e corrigidos para mesma massa de solo, em função da profundidade, conforme Sisti et al. (2004).

Para Costa et al. (2008) eAlves et al. (2008), em sistemas estáveis como a FN, a estabilidade dos estoques de $\mathrm{C}$ ocorre devido à adição contínua de resíduos orgânicos ao solo, sendo que o limite deste acúmulo é dependente da quantidade de resíduos produzidos e da ação microbiana, que tendem a ser estáveis, sofrendo alterações em situações específicas (secas, geadas, entre outras), mas cujos efeitos, pela periodicidade e/ou intensidade, normalmente, não se manifestam nos níveis de $\mathrm{C}$ estocados no solo.

No presente trabalho o solo da área $\mathrm{FN}$ foi considerado referência, sendo o estoque de $\mathrm{C}$ atual estimado em 107,67 $\mathrm{Mg} \mathrm{C}$ ha $^{-1}$, considerando o teor de $\mathrm{C}$ e a densidade de cada camada avaliada. Verifica-se redução de $28,09 \mathrm{Mg} \mathrm{C}^{-1}$ no solo em $\mathrm{PE}$ e $60,38 \mathrm{Mg} \mathrm{C} \mathrm{ha}^{-1} \mathrm{em} \mathrm{EM}$ em relação à $\mathrm{FN}$. Considerando os valores equivalentes em $\mathrm{CO}_{2}$ os valores atuais de estoque de $\mathrm{C}$ no solo e utilizando-se a mesma suposição de Diekow (2004) de que a perda de $\mathrm{C}$ durante o período tenha sido somente por oxidação microbiana, isso corresponde à emissão de $220,99 \mathrm{Mg} \mathrm{CO}_{2}$ ha $^{-1}$ do solo de EM para a atmosfera, no período após a retirada da vegetação nativa. Estes dados evidenciam os efeitos negativos que o manejo convencional (tradicional) pode promover na qualidade do solo, como da atmosfera, além de outros prejuízos ambientais, econômicos e sociais (DIEKOW et al., 2005). Por outro lado, não se pode afirmar que estes valores equivalem à perda real de $\mathrm{C}$ nas áreas, mas sim à condição atual de estoque de
$\mathrm{C}$, pois em PE diferentes manejos foram adotados após a retirada da FN.

Outros trabalhos realizados em solo de mata nativa em comparação a outras monoculturas florestais apresentam resultados distintos. Brun (2004) encontrou $112,6 \mathrm{Mg} \mathrm{C}^{-1} \mathrm{em}^{-1}$ mata secundária, em $60 \mathrm{~cm}$ de profundidade de solo. Para a espécie Acacia mearnsii, Caldeira et al. (2003) encontraram valores de 72,9 $\mathrm{Mg} \mathrm{C}$ ha $^{-1}$ e $69,5 \mathrm{Mg} \mathrm{C}$ ha $^{-1}$, em plantio de 4 e 6 anos, respectivamente, em profundidade de 0-60 cm. Rangel e Silva (2007) encontraram valores de $90,6 \mathrm{Mg} \mathrm{C}^{-1}$, em mata nativa e $105,28 \mathrm{Mg} \mathrm{C}^{-1}$, em eucalipto com 36 anos, para profundidade de $0-40 \mathrm{~cm}$. Os autores explicam que os maiores estoques de $\mathrm{C}$ em eucalipto provavelmente foram verificados devido ao maior adensamento do solo em comparação com a mata nativa.

Trabalhos no sul do Brasil, apesar de terem sido realizados sob florestas de espécies distintas, apresentam valores com elevada amplitude. Schumacher e Witschoreck (2004), em inventário de $\mathrm{C}$ em povoamentos de Eucalyptus sp. com oito anos, reportam estoque médio de 47,5 $\mathrm{Mg} \mathrm{C} \mathrm{ha}^{-1}$, na profundidade de 0-60 $\mathrm{cm}$. Na camada de 0-20 $\mathrm{cm}$, a média de $\mathrm{C}$ estocada foi de $25,8 \mathrm{Mg} \mathrm{C}^{-1}$, o que muito se assemelha a este estudo. Diferentes espécies resultam em diferentes estoques, Balbinot et al. (2003) avaliando o estoque de C em um plantio de Pinus taeda aos 5 anos de idade no Rio Grande do Sul, estimaram em $227,8 \mathrm{Mg} \mathrm{C}$ ha $^{-1}$, até a profundidade de $100 \mathrm{~cm}$ do solo, e concluíram que $65 \%$ deste total, ou seja, $147,8 \mathrm{Mg} \mathrm{C}^{-1}$ encontram-se nos primeiros $40 \mathrm{~cm}$, resultado notadamente superior ao deste estudo.

Resultados diferenciados encontrados na literatura possivelmente se devem às diferentes variações nas condições experimentais, como clima, tipo, preparo e manejo do solo e dos cultivos, idade das florestas que influenciam no crescimento das árvores e na decomposição dos resíduos depositados no solo (ZINN et al., 2002).

Em pastagens contíguas a área EM deste trabalho, Sordi et al. (2009) analisando as perdas de $\mathrm{C}$ do solo sob pastagem perene naturalizada (PPN) mantida por 60 anos, verificaram que a redução dos níveis de $\mathrm{C}$ foi de apenas $0,86 \mathrm{~g} \mathrm{~kg}^{-1}$, o que representa 3,42\% do $\mathrm{C}$ original. Esta pequena perda de $\mathrm{C}$ pode estar relacionada à baixa taxa de mineralização em PPN, onde não há mobilização de solo, dificultando o acesso dos microrganismos ao C. Além disto, argumentam que os sistemas radiculares das espécies presentes na PPN contribuem significati- 
vamente para o aporte de $\mathrm{C}$ ao solo. Em relação à pastagem cultivada com espécies anuais de verão e inverno (PCA), a redução foi de $3,2 \mathrm{~g} \mathrm{~kg}^{-1}$ na camada analisada $(0-50 \mathrm{~cm})$, representando $12,74 \%$ de $\mathrm{C}$ perdido, valor considerável, equivalente à emissão de 21,43 $\mathrm{Mg} \mathrm{CO}_{2}$ ha $^{-1}$ Salienta-se que esta perda foi observada em apenas dois anos de mobilização do solo, com a introdução da PCA na área. Ou seja, após 60 anos de utilização do solo com PPN e um estoque de 113,93 $\mathrm{Mg} \mathrm{C} \mathrm{ha}^{-1}$, em dois anos de uso do solo com uma pastagem implantada sob manejo intenso, verificaram significativa perda de $\mathrm{C}$, sendo mais representativa na camada arável do solo (0$20 \mathrm{~cm}$ ) com 13,35 $\mathrm{Mg} \mathrm{CO}_{2} \cdot$ ha $^{-1}$, já na camada de 20-50 $\mathrm{cm}$ a perda foi de $8,08 \mathrm{Mg} \mathrm{CO}_{2} \mathrm{ha}^{-1}$.

Para áreas de eucalipto, no momento da colheita das árvores, Pegoraro (2007) relata que o estoque de $\mathrm{C}$ na biomassa vegetal da parte aérea viva (cascas, folhas e galhos), foi em média, 10,6 $\mathrm{Mg} \mathrm{ha}^{-1}$. Esses resultados ressaltam a importância da manutenção dos resíduos da colheita na área de cultivo no momento do corte das árvores e, a não realização da prática da queima ou remoção dos resíduos após a colheita do lenho. No mesmo trabalho, verificou que a MOS foi o compartimento com o maior estoque total de $\mathrm{C}$ no ecossistema, com média de $53 \%$. Ao desconsiderar o que foi estocado pelo lenho do total do ecossistema, o que ocorre quando da colheita e exportação da parte comercial, e acrescer o que foi alocado às raízes e a MOS observou que 87,5\% do $C$ encontravam-se abaixo da superfície do solo, o que demonstra a grande importância deste compartimento na estocagem de $\mathrm{C}$.

Johnson e Curtis (2001) salientam a importância da manutenção da serapilheira em áreas com cultivo de eucalipto. Constataram incremento de 18 \% no estoque de C orgânico do solo quando, por ocasião da colheita, foi retirado apenas o tronco das árvores, por outro lado, verificaram uma redução de $6 \%$ quando foi efetuada a colheita da árvore inteira e a retirada dos resíduos da área.

Em um estudo realizado na condição do Cerrado Brasileiro, Zinn et al. (2002) verificaram que os solos sob povoamentos de eucalipto com sete anos de idade e sob vegetação nativa, apresentaram estoques de $\mathrm{C}$ de 41,64 e $65,75 \mathrm{Mg} \mathrm{C}^{-1}$, na camada de 0-60 cm, valores bem menores do que os verificados no presente trabalho. Além das condições já citadas, que podem afetar a manutenção da MOS e os estoques de $\mathrm{C}$ do solo (tempo de instalação da cobertura vegetal, tipo e condição inicial do solo, clima e práticas de manejo), neste caso, ao se com- parar estoques de $\mathrm{C}$ com o solo do Cerrado, provavelmente, a condição de temperaturas mais amenas do sul do Brasil, devem contribuir para uma mais lenta degradação da MOS e manutenção dos níveis de $\mathrm{C}$ no solo.

Sistemas de cultivo conservacionistas que privilegiam o aporte de resíduos e o menor revolvimento ou ausência de revolvimento do solo, garantem maior estabilidade dos sistemas (NEVES et al., 2004), promovem maior agregação do solo favorecendo maior estabilidade da MOS (PEDROSO et al., 2002) e podem mitigar a redução nos conteúdos de $\mathrm{C}$ e $\mathrm{N}$ do solo, causada por mudanças em sistemas naturais. Plantações de eucalipto estão inseridas neste contexto, como potenciais sequestradoras de $\mathrm{C}$ da atmosfera, pelo rápido crescimento, expressiva produção de biomassa e capacidade de capturar grande quantidade de $\mathrm{CO}_{2}$ da atmosfera, quando comparadas a outros ecossistemas, como florestas nativas clímax e cultivos agrícolas (PEGORARO, 2007).

Por outro lado, fazendo-se uma analogia com o relato de Cerri et al. (2008), quando reportam que alguns trabalhos indicam o aumento nos estoques de $\mathrm{C}$ no solo sob pastagem em relação aos solos de floresta, da mesma forma pode-se inferir para o aumento dos estoques de $\mathrm{C}$ do solo sob o plantio de eucalipto, considerando que esta conversão nem sempre é positiva em relação aos GEEs, em função do $\mathrm{C}$ oriundo da biomassa perdida pela floresta natural, ou emitido para a atmosfera, que em muitos casos é queimada. Estas transformações são ainda mais críticas quando se computam as perdas de partículas de solo (erosão) e da capacidade de armazenamento de água no solo, em função da falta de cobertura no solo e a perda da biodiversidade.

Considerando-se os dados e os argumentos até aqui apresentados, constata-se, como Spagnolo (2004), Diekow et al. (2005), Bayer et al. (2006) e Costa et al. (2008), que boas práticas de manejo, sem revolvimento do solo e com alto aporte de resíduos que promovam incrementos ou manutenção dos níveis de MOS devem ser indicadas, não somente como um meio de aumentar a produtividade dos cultivos, mas também como uma forma de reduzir os impactos adversos causados pela elevada concentração de GEEs na atmosfera Essas práticas podem conferir ao agricultor, no futuro, um benefício complementar, uma vez que as retirada de $\mathrm{CO}_{2}$ da atmosfera e sua retenção no solo (sequestro) poderão conferir reduções certificadas de emissão, negociáveis em bolsas de valores. Esse 
processo, Mecanismo de Desenvolvimento Limpo (MDL) é uma das opções já incluídas na ratificação do Protocolo de Quioto, para o cumprimento de metas de redução de emissão de países industrializados, apesar de que o compartimento solo só deve ser incluído como atividade elegível para obtenção de créditos de $\mathrm{C}$ a partir de 2013 (CERRI et al., 2008)

Desta forma, o Brasil poderá assumir uma posição privilegiada em relação a outros países que buscam reverter o processo de mudança climática global, tanto do ponto de vista das reduções de emissões, quanto do sequestro de $\mathrm{C}$, sendo imprescindíveis estudos que possibilitem mensurar as reais capacidades de estocagem de $\mathrm{C}$ dos solos, uma vez que poucos países possuem condições climáticas e tecnológicas apropriadas para a implantação de florestas e regeneração de áreas degradadas como o Brasil (ROCHA, 2002) e grandes extensões territoriais com aptidão agrícola e condições climáticas favoráveis ao crescimento vegetal e transferência de MO ao solo (CERRI et al., 2008).

Assim, como já relatado por Tornquist et al. (2005), os resultados obtidos são importantes para estudos, propostas e análise de políticas relacionadas à dinâmica do $\mathrm{C}$ e MOS em diferentes escalas (local, regional, entre outras), considerando que a estimativa dos estoques originais é um indicativo da capacidade de armazenagem de $\mathrm{C}$ nos solos. Em função das quantidades de $\mathrm{C}$ que armazena, o solo é um dos condicionantes de processos poluentes do ar, tendo em vista que a variação no estoque de $\mathrm{C}$ regula os teores desse elemento emitidos para a atmosfera (RANGEL e SILVA, 2007).

Finalizando, Amado et al. (2008) salientam que o sequestro de $\mathrm{C}$ em solos agrícolas é considerado um dreno lento, finito e com incertezas associadas a sua quantificação. No entanto, é uma alternativa economicamente viável, além de apresentar uma série de benefícios adicionais para a sociedade e para os agricultores.

\section{CONCLUSÕES}

Os teores de carbono são maiores nas camadas mais superficiais nos três segmentos, decrescendo em profundidade. Nestas camadas se verificam as maiores alterações dos estoques de carbono.

O solo da área de floresta natural apresenta o maior estoque de carbono, com 107,67 $\mathrm{Mg} \mathrm{ha}^{-1}$, seguido pelo plantio de eucalipto e plantio de erva-mate, com 79,58 e 47,29 $\mathrm{Mg} \mathrm{ha}^{-1}$, respectivamente.
Tomando a área de floresta natural como referência, verificam-se perdas de carbono no solo dos plantios de eucalipto e erva-mate, na ordem de 26,09 e $56,01 \%$, respectivamente.

Os resultados obtidos demonstram, claramente, que o manejo inadequado do solo, ou a alteração da cobertura vegetal (florestal), podem levar a grandes perdas do carbono estocado em um curto espaço de tempo. Desta forma, o solo, considerado um grande reservatório ou um dreno de carbono (fixação e estocagem), pode tornar-se uma grande fonte de carbono para a atmosfera, contribuindo para o aumento da concentração dos gases do efeito estufa na atmosfera terrestre.

\section{AGRADECIMENTOS}

À FAPESC e à FAPE/Unochapecó pelas bolsas e auxílio financeiro para o desenvolvimento do estudo.

\section{REFERÊNCIAS BIBLIOGRÁFICAS}

ADDISCOTT, T. M. Entropy and sustainability. European Journal of Soil Science, Exeter, v. 46, v. 1, p. 161-168, 1992.

ALVES, B. J. R. et al. Dinâmica de carbono em solos sob pastagens. In: SANTOS, G. de A. et al. (eds.). Fundamentos da matéria orgânica do solo: Ecossistemas tropicais e subtropicais. 2. ed. Porto Alegre: Metrópole, 2008. 654 p.

AMADO, T. J. C. et al. O solo agrícola e o mercado internacional de carbono. In: SANTOS, G. de A. et al. (eds.). Fundamentos da matéria orgânica do solo: ecossistemas, tropicais e subtropicais. Porto Alegre: Metrópole, 2008. p. 625-636.

ANDRADE, C. A. de. Fração orgânica de biossólidos e efeito no estoque de carbono e qualidade da matéria orgânica de um latossolo cultivado com eucalipto. 2004. 135 f. Tese (Doutorado em Agronomia) - Escola Superior de Agricultura "Luiz de Queiroz", Universidade de São Paulo, Piracicaba, 2004.

ARAUJO, M. A. et al. Propriedades Físicas de um Latossolo Vermelho Distrófico Cultivado e sob Mata Nativa. Revista Brasileira de Ciências do Solo, Viçosa, v. 28, p. 337-345, 2004.

AREVALO, L. A. et al. Metodologia para estimar o estoque de carbono em diferentes sistemas de uso da terra. Colombo, Embrapa Florestas, 2002. 41p.

BALBINOT, R. et al. Inventário do carbono 
orgânico em um plantio de Pinus taeda aos 5 anos de idade no Rio Grande do Sul. Ciências Exatas e Naturais, Curitiba, v. 5, n. 1, p. 59-68, 2003.

BAYER, C. et al. A method for estimating coefficients of soil organic matter dynamics based on long-term experiments. Soil and Tillage Research, Amsterdan, v. 91, p. 217-226, 2006.

BAYER, C. et al. Armazenamento de carbono em frações lábeis da matéria orgânica de um Latossolo Vermelho sob plantio direto. Pesquisa Agropecuária Brasileira, Brasília, v. 39, n. 7, p. 677-683, 2004.

BRUN, E. J. Biomassa e nutrientes na floresta decidual, em Santa Tereza, RS. 2004. 136 f. Dissertação (Mestrado em Engenharia Florestal) Universidade Federal de Santa Maria, Santa Maria, 2004.

CALDEIRA, M. V. et al. Determinação de carbono orgânico em povoamentos de Acacia mearnsii De Wild. plantados no Rio Grande do Sul. Revista Acadêmica: Ciências Agrárias e Ambientais, Curitiba, v. 1, n. 2, p. 47-54, 2003.

CAVENAGE, A. et al. Alterações nas propriedades físicas de um Latossolo Vermelho-Escuro sob diferentes culturas. Revista Brasileira de Ciência do Solo, Viçosa, v. 23, n. 4, p. 997-1003, 1999.

CERRI, C. C. et al. O balanço de carbono nos solos tropicais. In: REUNIÃO BRASILEIRA DE FERTILIDADE DO SOLO E NUTRIÇÃO DE PLANTAS, 28., 2008. Londrina. Anais... Londrina: SBCS-UEL. 2008. 1 CD Rom.

COSTA, F. de S. et al. Aumento de Matéria Orgânica num Latossolo Bruno em Plantio Direto. Ciência Rural, Santa Maria, v. 34, n. 2, p. 587-589, 2004. COSTA, G. S. et al. Decomposição e liberação de nutrientes da serapilheira foliar em povoamentos de Eucalipyptus grandis no Norte Fluminense. Revista Árvore, Viçosa, v. 29, n. 4, p. 563-570, 2005.

COSTA, F. de S. et al. Estoque de carbono orgânico no solo e emissões de dióxido de carbono influenciadas por sistemas de manejo no Sul do Brasil. Revista Brasileira de Ciência do Solo, Viçosa, v. 32, n. 1, p. 323-332, 2008.

DIEKOW, J. et al. Sistemas conservacionistas de preparo do solo e implicações no ciclo do carbono. São Carlos: Embrapa, 2004. 18 p. (Documento 12.)

DIEKOW, J. et al. Soil $\mathrm{C}$ and $\mathrm{N}$ stocks as affected by cropping systems and nitrogen fertilization in a southernBrazil Acrisol managed under no-tillage for 17 year. Soil and Tillage Research, Amsterdan, v. 81, p. 87-95, 2005.
EMBRAPA. Centro Nacional de Pesquisa de solo. Manual de Métodos de análise de Solo. 2. ed. Rio de Janeiro: Embrapa Solos. 1997. 212 p.

EMBRAPA. Centro Nacional de Pesquisa de Solos. Sistema brasileiro de classificação de solos. 2. ed. Rio de Janeiro, 2006. 306 p.

GAMA-RODRIGUES, E. F. da et al. Alterações na biomassa e na atividade microbiana da serapilheira $\mathrm{e}$ do solo, em decorrência da substituição de cobertura florestal nativa por plantações de eucalipto, em diferentes sítios da região Sudeste do Brasil. Revista Brasileira de Ciência do Solo, Viçosa, v. 32, p. 1489-1499, 2008.

IPCC - INTERGOVERNMENTAL PAINEL ON CLIMATE CHANGE. Climate Change 2001. Synthesis report. The scientific basis. Cambridge: Cambridge University Press, 2002. 397 p.

JOHNSON, D. W. ; CURTIS, P. S. Effects of forest management on soil $\mathrm{C}$ and $\mathrm{N}$ storage: meta analysis. Forest Ecology and Management, Victoria, v. 140, n. 2-3, p. 227-238, 2001.

MINISTÉRIO DA CIÊNCIA E TECNOLOGIA. Coordenação-Geral de Mudanças Globais do Clima. Segunda Comunicação Nacional do Brasil à Convenção-Quadro das Nações Unidas sobre Mudança do Clima. Brasilia. 2010. 280 p.

MUELLER - DOMBOIS, D.; ELLENBERG, H. Airms and methods of vegetation ecology. New York: John Wiley \& Sons, 1974. 547 p.

NEVES, C. M. N. et al. Estoque de Carbono em sistemas agrossilvopastoril, pastagem e eucalipto sob cultivo convencional na Região Noroeste do Estado de Minas Gerais. Ciência Agrotécnica, Lavras, v. 28, n. 5, p. 1038-1046, 2004.

PANDOLFO, C. Atlas climatológico digital de Estado de Santa Catarina. 2001. Disponível em: http://ciram.epagri.sc.gov.br/portal/ Controlador?url=jsp/biblioteca/biblioteca_lista.js p\&command $=$ ExibirPublicacoes $\&$ module $=$ websi te\&tipo=biblioteca\&st_publ=1 Acesso em: 13 de outubro de 2011.

PEDROSO, M. T. et al. Estoques de $\mathrm{C}$ e $\mathrm{N}$ em diferentes sistemas de manejo do solo na microbacia de Passo do Meio, Cristal - RS. Os (des) Caminhos do Uso da Água na Agricultura Brasileira. In: REUNIÃO BRASILEIRA DE MANEJO E CONSERVAÇÃO DO SOLO E DA ÁGUA, 14., 2002. Cuiabá. Anais... Cuiabá: SBCS. 2002. 1 CD Rom.

PEGORARO, R. F. Seqüestro de carbono e alterações bioquímicas da matéria orgânica de solos cultivados com eucalipto. $2007.151 \mathrm{f}$. Tese 
(Doutorado em Solos e Nutrição de Plantas) Universidade Federal de Viçosa, Viçosa, 2007.

PICCOLO, G. A. et al. Changes in soil organic matter under different land management in Misiones Province (Argentina). Scientia Agricola, Piracicaba, v. 65, n. 3, p. 290-297, 2008.

RANGEL, O. J. P.; SILVA, C. A. Estoques de carbono e nitrogênio e frações orgânicas de Latossolo submetido a diferentes sistemas de uso e manejo. Revista Brasileira de Ciência do Solo, Viçosa, v. 31, n. 6, p. 1609-1623, 2007. ROCHA, M. T. O aquecimento global e os instrumentos de mercado para a solução do problema. In: SANQUETA, C. R. et al. (ed.). As florestas e o carbono. Curitiba: 2002. p. 1-34.

ROCHA, M. T. O aquecimento global e os instrumentos de mercado para a solução do problema. In: SANQUETA, C. R. et al. (ed.). As florestas e o carbono. Curitiba: 2002. p. 1-34.

SPAGNOLLO, E. Dinâmica da matéria orgânica em agroecossistemas submetidos à queima $\mathrm{e}$ manejos dos resíduos culturais. 2004. 210 f. Tese (Doutorado em Ciência do Solo) - Universidade Federal de Santa Maria, Santa Maria

SCHOLES, R. J.; NOBLE, I. R. Climatic Change: Storing Carbon on Land. Science, Washington, v. 294, n. 5544, p. 1012-1013, 2001.

SCHUMACHER, M. V. et al.. Quantificação de carbono orgânico na serapilheira, sub-bosque e solo de uma Floresta de Pinus elliottii Engelm. aos 36 anos, em Santa Maria, RS. In. SANQUETTA, C. R. et al. (eds.). Fixação de carbono: atualidades, projetos e pesquisas. Curitiba, 2004. p. 125-132.

SCHUMACHER, M. V.; WITSCHORECK, R. Inventário de carbono em povoamentos de
Eucalyptus ssp. nas propriedades fumageiras do Sul do Brasil: um estudo de caso. In. SANQUETTA, C. R. et al (eds.). Fixação de carbono: atualidades, projetos e pesquisas. Curitiba, 2004. p. 111-124.

SISTI, C. P. J. et al. Change in carbon and nitrogen stocks in soil under 13 years of conventional or zero tillage in southern Brazil. Soil Tillage Research., Amsterdan, v.76, p. 39-58, 2004.

SORDI, A. et al. Estoque de carbono do solo, sob diferentes sistemas de uso da Terra. In: CONGRESSO BRASILEIRO DE CIÊNCIAS DO SOLO, 32., 2009, Fortaleza. Anais... Fortaleza: SBCS. 1 CD-Rom.

SOUZA, I. M.Z. Carbono e nitrogênio da biomassa microbiana do solo em áreas reflorestadas comparadas ao campo e mata nativa no planalto dos campos gerais, SC. 2005. 50 f. Dissertação (Mestrado em Ciência do Solo) - Universidade do Estado de Santa Catarina, Lages, 2005.

SUZUKI, L.E.A. S. et al. Alterações de propriedades físicas em Latossolo Vermelho amarelo do Noroeste Paulista sob três condições de uso e manejo. In: CONGRESSO BRASILEIRO DE ENGENHARIA AGRÍCOLA, 31., 2002. Salvador. Anais... Salvador: UFRB-CONBEA, 1 CD Rom.

TORNQUIST, C. G. et al. Estoques Originais de Carbono Orgânico em Solos do Planalto do Rio Grande do Sul. In: CONGRESSO BRASILEIRO DE CIÊNCIA DO SOLO, 30., 2005. Viçosa. Anais.... Viçosaq: SBCS-UFV, 1 CD Rom.

ZINN, Y. L. et al. Soil organic carbon as affected by afforestation with Eucalyptus and Pinus in the Cerrado region of Brazil. Forest Ecology and Management, Victoria, v. 166, p. 285-294, 2002. 